\title{
Processing of Immunoisolated Pancreatic Islets: Implications for Histological Analyses of Hydrated Tissue
}

BioTechniques 32:612-619(March 2002)

\author{
Bart J. De Haan, Harry van \\ Goor, and Paul De Vos \\ University of Groningen, \\ Groningen, The Netherlands
}

\begin{abstract}
Routine tissue processing is usually associated with histological artifacts as a consequence of shrinkage and distortion during dehydration required for embedding. With hydrated specimens such as lung, embryon$i c$, and tissues in hydrophilic membranes, tissue processing can induce severe artifacts that interfere with adequate microscopic evaluation. Here we present a method for embedding hydrophilic alginate-polylysine microencapsulated pancreatic tissue that combines the absence of histological artifacts with a practical tissue processing method. We found that the glycol-methacrylate (GMA)-embedding method preserved the integrity of the encapsulated tissue better than snap-freezing or paraffin embedding, but the overall quality of the hydrophilic capsules remained poor. Next, we modified the GMA method by introducing gradual dehydration to investigate whether the integrity of the sectioned capsules was better maintained by a more gradual pattern of water extraction. This modification resulted in well-preserved morphological details of the hydrophilic membranes, hydrogel-cell interface, and encapsulated pancreatic tissue. Subsequent routine staining gave excellent contrast between the islet tissue and hydrophilic components, which allowed adequate quantitative histological and pathological comparisons.
\end{abstract}

\section{INTRODUCTION}

Encapsulation in semipermeable and immunoprotective artificial membranes allows for successful transplantation of allo- and xenogenic tissues in the absence of immunosuppression $(4,14,16,17)$. Transplantation of encapsulated hormone-producing cells is under investigation for a wide variety of diseases, including anemia (11), liver failure (21), pituitary disorders (2), central nervous system insufficiencies (1), and diabetes (13).

Conventional light microscopy is commonly used to determine the biocompatibility of the artificial mem branes and for investigating the integrity of the encapsulated biological material. Unfortunately, this is not without problems since processing of bioartificial tissue is usually associated with severe histological artifacts $(8,12)$ as a consequence of shrinkage and distortion during the processing required for embedding.

When hydrophilic material is used in the design of the artificial membranes, shrinkage-induced histological artifacts can even interfere with adequate microscopic evaluation. This has been noticed by several authors working with alginate-polylysine (APA) capsules as commonly used for immunoprotection of pancreatic islets $(8,10$, 12). A number of modified paraffin (15) and resin (8) embedding methods have been proposed for studies with APA capsules, but none combines the absence of histological artifacts with a practical method of processing.
In the present study, we have selected the most appropriate embedding methods for testing modifications by comparing three commonly used processing methods [i.e., snap-freezing, paraffin embedding, and glycol methacrylate (GMA) resin embedding]. Next, we have investigated whether modifications in the selected embedding method for APA-encapsulated islet tissue can reduce shrinkage-induced artifacts. Subsequently, we have analyzed the preservation of morphological details for histochemical assessment.

\section{MATERIALS AND METHODS}

\section{Islet Isolation and Graft Recipients}

Male inbred Albino Oxford (AO/G) rats were obtained from the Central Animal Laboratory of Groningen. $\mathrm{AO} / \mathrm{G}$ rats weighing 300-350 g served as donors. AO/G rats weighing 290$320 \mathrm{~g}$ were used as recipients of islet grafts. Diabetes was induced in these rats by injection of 75-90 mg/kg Streptozotocin (Zanosar; Upjohn, Kalamazoo, MI, USA) via the tail vein.

Islets were isolated as previously described (19). Briefly, after surgical removal from the abdomen, the pancreas was chopped and digested with 1.0 and $0.7 \mathrm{mg} / \mathrm{mL}$ collagenase (Sigma type XI; Sigma, St. Louis, MO, USA) using a two-stage incubation of $20 \mathrm{~min}$ at $37^{\circ} \mathrm{C}$. Islets were separated from exocrine tissue using a discontinuous dextran gradient (18) and further purified by handpicking. The total islet volume 
obtained by the isolation procedure was determined by measuring the diameters of islets in a $4 \%$ aliquot of the islet suspension. In each transplant experiment, we collected 3500-4200 islets, which corresponds to $10-15 \mu \mathrm{L}$ islet tissueapproximately equal to the islet volume of one normal pancreas.

Islets were cultured before encapsulation to reduce contamination of the islets with exocrine tissue, ducts, or damaged cells. They were cultured in untreated petri dishes (Greiner, Alphen a/d Rijn, The Netherlands) in portions of 100 islets per $25 \mathrm{~cm}^{2}$ for $19-44 \mathrm{~h}$ in CMRL1066 [containing 10\% FCS (Invitrogen, Groningen, The Netherlands), $8.3 \mathrm{mM}$ glucose, $10 \mathrm{mM}$ HEPES, $\mathrm{pH}$ 7.4 , and $1 \%$ Penicillium/Streptomycin] at $37^{\circ} \mathrm{C}$ in humidified air containing $5 \% \mathrm{CO}_{2}$.

\section{Alginates}

Low-guluronic acid (G) (Manucol), intermediate-G (Keltone LV), and high$\mathrm{G}$ (Manugel) sodium alginates were obtained from Kelco International (London, UK). Crude sodium alginate (Keltone LV; Kelco International) was purified as previously described (4).

Purified alginates were dissolved at $4^{\circ} \mathrm{C}$ in Krebs-Ringer-HEPES (KRH) with an appropriate osmolarity to a solution with a viscosity of $4 \mathrm{~cm}^{2} / \mathrm{s}$. The viscosity of an alginate solution is determined by the concentration of alginate, but different alginates have different viscosities. This value implies a concentration of $4.3 \%$ for the low-G solution, a $3 \%$ concentration for the intermediate-G solution, and a $2 \%$ concentration for the high-G solution. Before application, the solutions were sterilized by $0.2 \mu \mathrm{m}$ filtration. For the initial experiments to overcome the histological artifacts, we used intermediate- $\mathrm{G}$ alginate since this is the type of alginate we routinely apply for in vivo studies.

\section{Encapsulation}

After culture, islets were washed 3-5 times with RPMI containing 10\% FCS and subsequently suspended in sterile filtered $(0.2 \mu \mathrm{m}$ filtration $)$ purified alginate solution. The alginate solution was converted into droplets using an air-driven droplet generator as previously described (3). Polylysine-alginate encapsulation was performed as described elsewhere (9). Briefly, the alginate droplets were transformed to alginate beads by gelling in a $100 \mathrm{mM}$ $\mathrm{CaCl}_{2}, 10 \mathrm{mM}$ HEPES, pH 7.4, 2 mM $\mathrm{KCl}$ solution for at least $5 \mathrm{~min}$. After gelation, the beads had a diameter of 450-550 $\mu \mathrm{m}$. Subsequently, the Ca-alginate beads were suspended for $1 \mathrm{~min}$ in $\mathrm{KRH}$ containing $2.5 \mathrm{mM} \mathrm{CaCl}_{2}$. A polylysine membrane was formed by suspending the alginate beads in $0.1 \%$ polylysine solution for $10 \mathrm{~min}$ (polylysine- $\mathrm{HCl}$, molecular weight $22 \mathrm{kDa}$; Sigma). Non-bound polylysine was removed by three successive washes for 3 min with Ca-free KRH containing 135 $\mathrm{mM} \mathrm{NaCl}$. The outer alginate layer was subsequently applied by a 5 -min incubation in $10 \times$ diluted alginate solution. Finally, the capsules had a diameter of 700-800 $\mu \mathrm{m}$.

Capsules were injected into the peritoneal cavity. Upon peritoneal lavage, microcapsules were either freely floating and non-adherent or adherent to the surface of abdominal organs. First, nonadherent microcapsules were retrieved by peritoneal lavage and brought into a syringe with appropriate measures for quantification of the retrieval rate (4).

All animal experiments were performed according to the institutional council guidelines.

\section{Tissue Processing}

Initial experiments were performed with freshly encapsulated islets without prior implantation. Snap-frozen material was prepared by embedding APA microencapsulated islets in tissue-tek matrix (Tissue-Tek ${ }^{\circledR}$; Sakura Finetek Europe, Zoeterwoude, The Netherlands), followed by immediate freezing in pre-cooled 2-methylbutane. Five-micron sections were prepared using a Leica CM 1850 microtome (Wetzlar, Germany) [a Reichert-Jung 2055 microtome (Nusslook, Germany) was used for GMA sectioning].

For GMA embedding, microencapsulated islets were fixed for $3 \mathrm{~h}$ in $2 \%$ paraformaldehyde at $4^{\circ} \mathrm{C}$, buffered with $0.1 \mathrm{M}$ phosphate $(\mathrm{pH} 7.2)$. This was found to be an adequate fixation procedure in a previous study (8). After fixation, microencapsulated islets were 
washed overnight at $4^{\circ} \mathrm{C}$ in $\mathrm{PBS}(\mathrm{pH}$ 7.2) containing $6 \%$ sucrose. The sample was then dehydrated in $100 \%$ acetone at $4^{\circ} \mathrm{C}$ for $1 \mathrm{~h}$ (sudden dehydration). Next, Technovit 8100 solution A (Heraeus Kulzer, Friedensdorf, Germany) was added and allowed to infiltrate the tissue for $6 \mathrm{~h}$ at $4^{\circ} \mathrm{C}$. A $1: 30 \mathrm{di}$ lution of solution $\mathrm{B}$ was then added to harden the Technovit. Excess embedding medium was aspirated, and the encapsulated islets and block holders were placed in the embedding molds. Paraffin was poured around the block holders to prevent disturbance of the polymerization by oxygen present in atmospheric air. Two-micron sections were then prepared.

For paraffin embedding, encapsulated islets were fixed for $3 \mathrm{~h}$ in Bouin's solution since we found that this fixative was more adequate for evaluation of microencapsulated islets in paraffin than $2 \%$ paraformaldehyde. The samples were dehydrated by sequential incubation in $80 \%, 90 \%, 96 \%$, and $100 \%$ ethanol (sudden and conventional dehydration). Samples were then treated with isoamyl acetate, amylacetate celloïdine, and embedded in paraffin wax at $65^{\circ} \mathrm{C}$. Five-micron sections were then prepared. Before histochemical or immunocytochemical analyses, the sections were deparaffinized by two 5-min incubations in xylene. Sections were then incubated in $100 \%, 96 \%, 90 \%, 70 \%$, and $50 \%$ ethanol and water for 2 min each and finally stored in PBS until use.

In a number of experiments, we studied the morphology of encapsulated islets after gradual dehydration instead of sudden dehydration of samples before embedding in GMA or paraffin. For paraffin embedding, gradual dehydration of fixed samples began in a $25 \%$ ethanol solution instead of an $80 \%$ ethanol solution. During gradual dehydration, the ethanol concentration was increased in steps of 5\% until the sam ples were completely dehydrated in a $100 \%$ ethanol solution. For GMA embedding, the fixed samples were dehydrated in graded concentrations of ethanol, acetone, or GMA monomers using identical starting concentrations and the same stepwise concentration increase as used for gradual dehydration of paraffin-embedded tissues. During gradual dehydration, the integrity of the capsules was studied by using a dissection microscope with a fluorescent illuminator (BVB-125 and 31-3366; Bausch \& Lomb, Schiphol-Rijk, The Netherlands).

\section{Histochemistry and Immunohistochemistry}

A panel of staining procedures was used: hematoxylin and eosin $(\mathrm{H} \& \mathrm{E})$, Loeffler's methylene blue, Romanovsky-Giemsa, and Celestin blue.

Before immunostaining of GMAembedded tissue, sections were dried at $37^{\circ} \mathrm{C}$ and incubated in $0.01 \%$ trypsin (in $6.8 \mathrm{mM} 0.1 \% \mathrm{CaCl}_{2}$ and $0.1 \mathrm{M}$ Tris-HCl, $\mathrm{pH} \mathrm{7.8)} \mathrm{for} 5 \mathrm{~min}$. For im munostaining in $\mathrm{PBS}$, the sections were incubated for $2 \mathrm{~h}$ at $37^{\circ} \mathrm{C}$ with guinea pig anti-insulin antibody [(Dako Diagnostics BV, Glostrup, Denmark), 1:250 dilution in PBS $+1 \%$ BSA]. After several washes in PBS ( $\mathrm{pH} 7.2$ ), sections were incubated with $0.075 \% \mathrm{H}_{2} \mathrm{O}_{2}$ in PBS, followed by an incubation of 30 min at $20^{\circ} \mathrm{C}$ to block endogenous peroxidase. Next, a 1:40 dilution goat antirabbit horseradish peroxidase-conjugated second step reagent (Dako Diagnostics BV) diluted in PBS ( $\mathrm{pH}$ $7.2)+1 \%$ BSA supplemented with $5 \%$ normal rat serum was added, and sections were incubated for $45 \mathrm{~min}$ at $20^{\circ} \mathrm{C}$. Peroxidase activity was demonstrated by applying $0.2 \mathrm{mg} / \mathrm{mL} \mathrm{3-}$ amino-9-ethylcarbazole (AEC) containing hydrogen peroxide.

\section{RESULTS}

Initial experiments were performed with freshly encapsulated islets without prior implantation. A comparative study of three processing techniques (i.e., snap-freezing, paraffin embedding, and GMA embedding) showed that processing and embedding in GMA or paraffin preserved both the capsule and the tissue in intermediate- $G$ alginates better than snap-freezing (Figure 1). Also, snapfreezing was associated with a significant loss of encapsulated islets during staining, which could not be prevented by coating of the microscope slides. However, irrespective of the method, the overall preservation and integrity of the hydrogel membrane remained poor. Be- cause these types of artifacts are usually interpreted to be the consequence of shrinkage induced by dehydration (8), we investigated whether the integrity of the sectioned capsules was better maintained by a more gradual pattern of water extraction. This was done for encapsulated islets embedded in GMA and in paraffin.

\section{Effects of Gradual Dehydration}

When in the paraffin-embedding procedure microencapsulated islets were subjected to gradual instead of sudden dehydration conditions, we found some improvement in both the morphology of the capsule-membrane and the preservation of the islet tissue. However, the overall quality of the sections remained unsatisfactorily low since the membranes were still severely folded.

In the GMA-embedding method, gradual dehydration instead of sudden dehydration gave better results, as evidenced by the preservation of the integrity of the capsules and by the preservation of the morphology of the encapsulated tissue (Figure 2). This positive effect of gradual dehydration was not restricted to diluted acetone but was also observed when diluted acetone was replaced by diluted GMA monomers or when diluted acetone was replaced by diluted ethanol.

The efficacy of ethanol as a gradual dehydrant for GMA embedding is surprising since it could not prevent folding of the membrane of encapsulated islets embedded in paraffin. This might be associated with the use of different fixatives for paraffin and for GMA embedding (e.g., Bouin's solution for paraffin embedding and $2 \%$ paraformaldehyde for GMA embedding) since it cannot be excluded that fixation in Bouin's solution induces folding of the membranes already before the gradual dehydration step in the paraffin-embedding procedure. Therefore, in a separate series of experiments, we compared the degree of distortion in paraffin-embedded microencapsulated islets that were fixed in Bouin's solution or in $2 \%$ paraformaldehyde. We found that the distortion and, thus, the inefficacy of ethanol as a gradual dehydrant in paraffin embedding is related to other pertinent differences between 


\section{Research Report}

paraffin and GMA embedding since both Bouin's and 2\% paraformaldehyde-fixed samples embedded in paraffin contained large numbers of artifacts.

\section{Histochemistry}

Since our modifications were efficacious in preventing the distortion of the encapsulated tissue in the GMA-em bedding method, but not in the paraffinembedding method, we restricted our subsequent investigations to encapsulated tissue embedded in GMA.

The gradual dehydration procedure does not interfere with the ability to perform histochemistry on GMA-embedded microencapsulated islets. Sections of gradually or suddenly GMA-embed- ded microencapsulated islets were indistinguishable when stained by Gill's H\&E, Loeffler's methylene blue, Romanovsky-Giemsa, and Celestin blue. However, we found that H\&E and Loeffler's methylene blue were the only stains that provided enough contrast between the islets and the hydrophilic membrane to allow for detailed study of the membrane-tissue interface.

\section{Immunohistochemistry}

To investigate whether gradual dehydration interferes with the antigenicity of the tissue, we have immunostained sections of gradually dehydrated GMA-embedded microencapsulated islets for insulin. Sections of suddenly dehydrated GMA-embedded microencapsulated islets were used as control.

Both gradually and suddenly dehydrated GMA-embedded sections can be readily stained for insulin. However, the staining intensity was more pronounced in sections of capsules subjected to sudden dehydration than in sections subjected to gradual dehydration. This difference in staining intensity did not interfere with adequate assessment of the sections.

\section{Effect of Type of Alginate}

Alginates are composed of mannuronic acid $(\mathrm{M})$ and guluronic acid (G). There are different types of alginates available with varying $\mathrm{G}$ content.

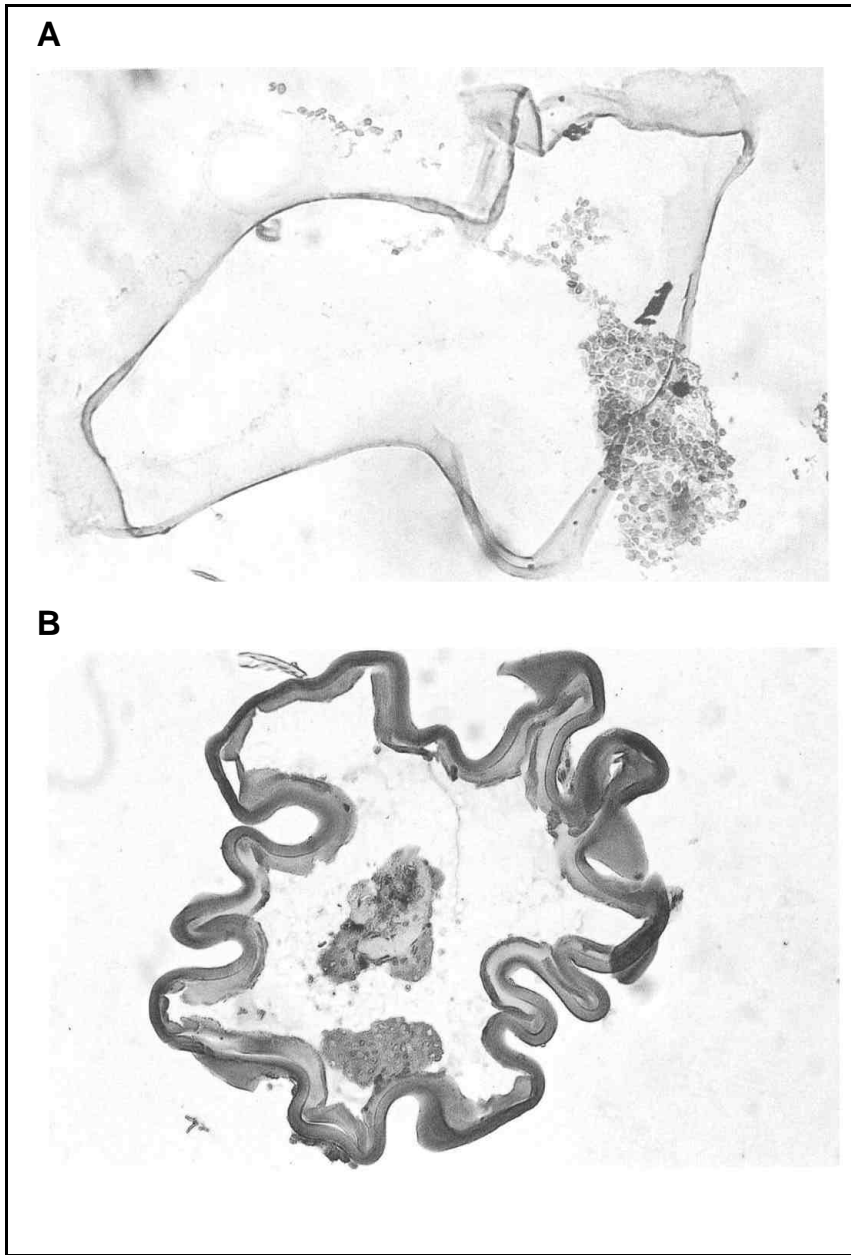

Figure 1. Alginate-polylysine-microencapsulated islets processed according to two histological techniques. (A) A section of snap-frozen encapsulated islets. (B) A section of paraffin-embedded encapsulated islets. The embedding procedures were performed according to the standard methods described in the Materials and Methods section. All slices are stained with H\&E. Original magnification, 200x.

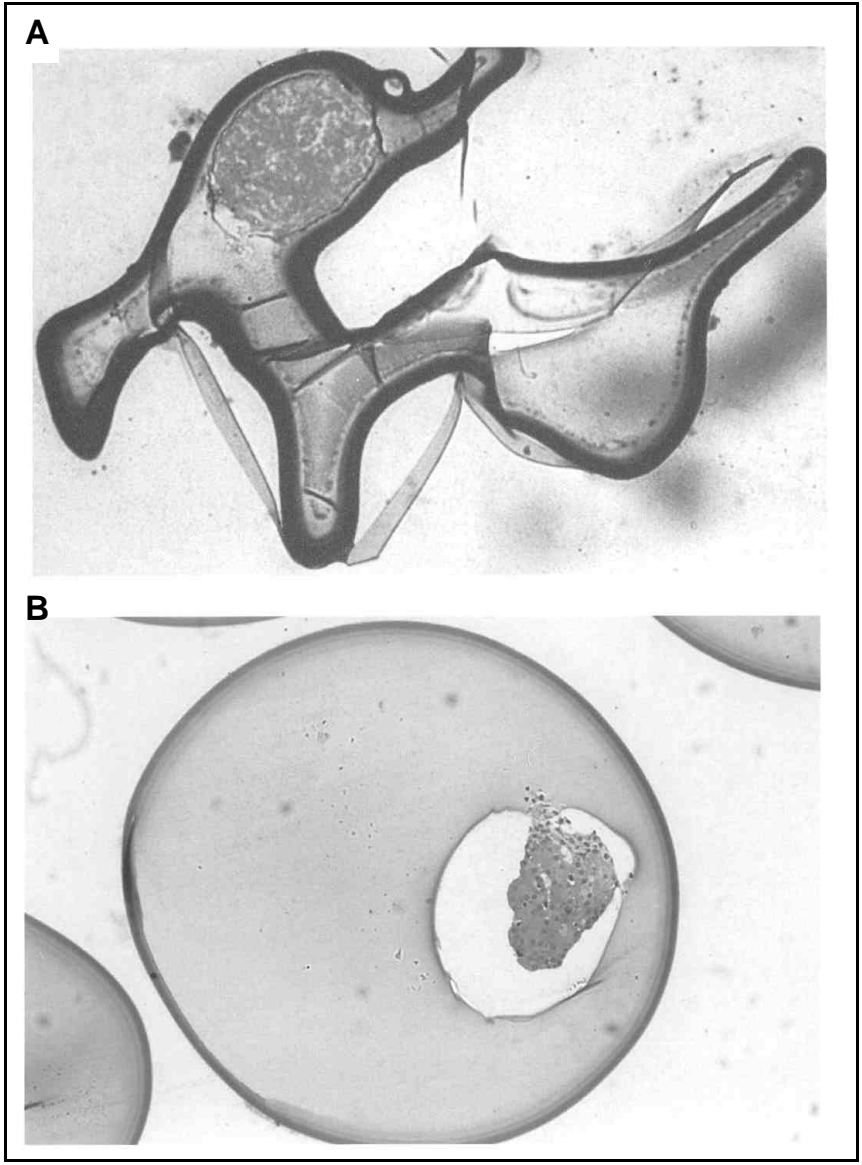

Figure 2. The effect of gradual dehydration of alginate-polylysine capsules. (A) An encapsulated islet processed according to the GMA procedure with sudden dehydration. Note the severe folding of the capsule membrane. (B) Spherical encapsulated islets after gradual dehydration of the capsules. Note the empty space between the islet and the alginate, which is caused by shrinkage of the islets after encapsulation. Also note the clear distinction between the outer alginate-polylysine membrane and the inner alginate core surrounding the islet. All slices are stained with Loeffler's methylene blue. Original magnification, $200 x$. 
Since in encapsulation research different types of alginate are used, we questioned whether the new processing method could also be applied on capsules prepared with low-G or high-G alginates instead of on capsules prepared of intermediate- $G$ alginates.

The type of alginate did not appear to influence the quality of the sections processed according to the gradual dehydration method since, irrespective of the type of alginate, we found an absence of distortion and an adequate quality of the membrane-tissue interface.

\section{Evaluation of Encapsulated Islets after Implantation}

All streptozotocin-induced diabetic $\mathrm{AO} / \mathrm{G}$ recipients of encapsulated allogenic islets became normoglycemic within five days of implantation. One of the seven recipients failed within 10 days. This unsuccessful recipient was sacrificed, and capsules were retrieved by peritoneal lavage. The six other, successful grafts were retrieved by peritoneal lavage at four weeks after implantation, and most of the capsules were freely floating in the abdominal cavity without adhesion to the abdominal wall or organs, as quantified by a retrieval rate of more than $90 \%$ of the capsules.
The retrieved capsules were processed according to the gradual dehydration procedure for GMA embedding.

The mean percentage of capsules with cellular overgrowth was usually well below $10 \%$, with the exception of the animal with the failing graft at 10 days after implantation. Virtually all capsules were overgrown in this unsuccessful graft.

Histological slides were used to evaluate the causes of the cellular overgrowth. Figure 3 shows a classical example of a capsule with a localized defect surrounded by inflammatory cells invading the capsule and the allogenic islet. In the unsuccessful graft, we found groups of bacteria (Figure 4), which suggest a bacterial infection to be the cause of the early graft failure.

\section{DISCUSSION}

Our study demonstrates that GMA embedding is better than snap-freezing or paraffin embedding for microscopic evaluation of alginate-polylysine microencapsulated islets. This method was not associated with the loss of em bedded material during staining and it was the only method that could be modified to preserve the integrity of the capsules and the morphology of the encapsulated tissue.

Gradual dehydration proves to be mandatory for preserving the integrity of the capsules since the osmotic shift of water out of the capsule core during sudden dehydration induces severe capsule deformation. As a consequence, hydrogel-tissue interfaces of capsules are distorted and membranes are broken, which interferes with adequate histological evaluation.

Histochemistry could be adequately performed on slices of gradually dehydrated GMA-embedded islets. We now routinely use Loeffler's methylene blue since it allows optimal contrast between tissues and the capsule membranes. The sharp purple metachromatic staining (8) of the alginate-polylysine membrane allows for the meticulous inspection of the integrity of the capsule membrane and for the detection of inadequacies associated with early graft failure such as tails on the beads $(5,6,20)$ and broken membranes. Also, it allows one to study the interaction and type of cells surrounding the membrane after retrieval from the implantation site $(6,7,17)$.

Our conclusion that paraffin embedding is inadequate for processing encapsulated islets does not corroborate the findings of Lue et al. (12), who

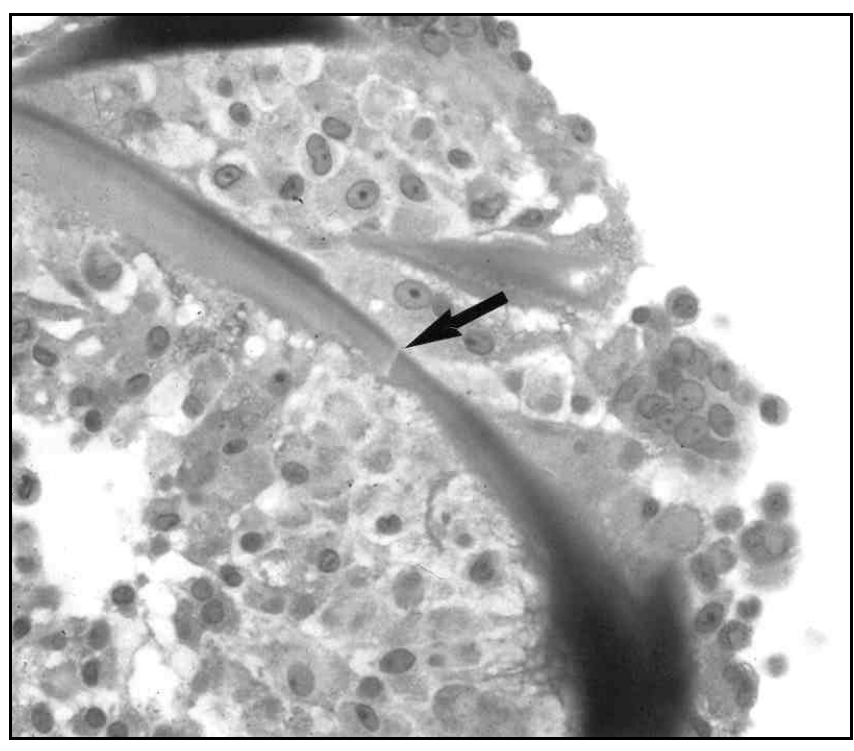

Figure 3. A localized defect in an alginate-polylysine capsule containing an allogenic islet. Note the optimal morphology of the capsule membrane and the clear distinction between the alginate-polylysine membrane and the inflammatory cells (arrow). All slices are stained with Loeffler's methylene blue. Original magnification, $250 \times$.

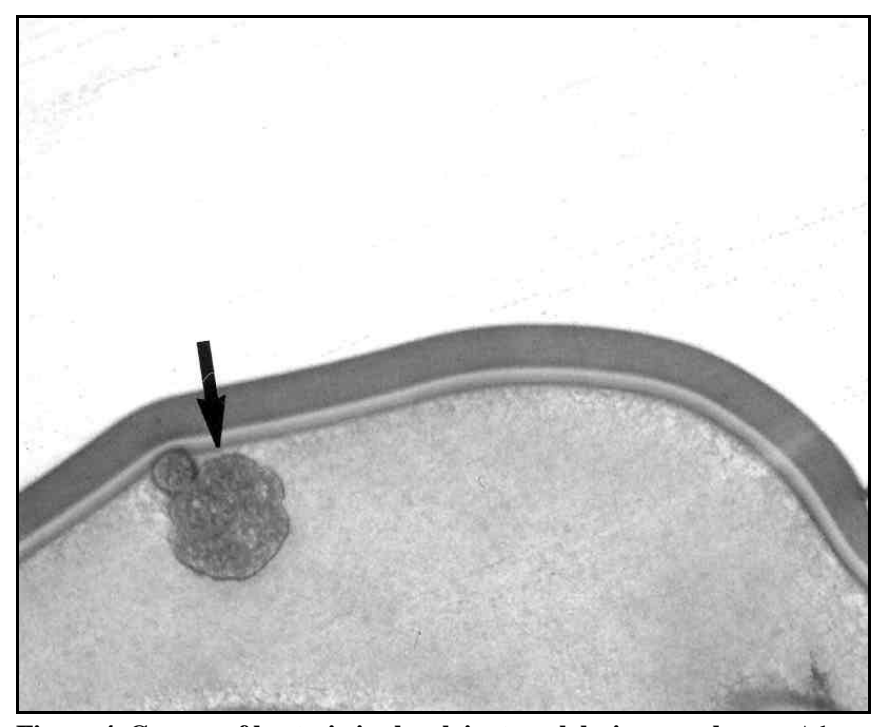

Figure 4. Groups of bacteria in the alginate-polylysine membrane. A bacterial infection caused failure of the graft. Bacteria (arrows) could be identified in histological slices of the capsules. Note the optimal morphology of the capsule membrane and the clear distinction between the alginate and the groups of bacteria. All slices are stained with Loeffler's methylene blue stained. Original magnification, $250 \times$. 
show that distortion of paraffin-embedded encapsulated tissue can be prevented by using gradual solvent and medium exchange. However, these authors used specially designed equipment for gradual solvent exchange in steps of $2 \%$, which might be associated with less distortion than with the graded solution exchange in steps of $5 \%$ in the present study. Unfortunately, the equipment for gradual solvent exchange is complicated, labor intensive, and impractical for the routine processing of encapsulated islet tissue. GMA embedding with gradual dehydration gives similar results and does not require any sophisticated equipment.

Our observation that standard GMA embedding without gradual dehydration is not suitable for microscopic examination of encapsulated pancreatic islets appears to contradict the conclusion of our previous study in which we found that the GMA-embedding method with standard, rapid dehydration was adequate for processing of alginate-polylysine-encapsulated islets. That study, however, was performed with capsules with a high degree of permeability and no immunoprotective properties since they were prepared of $1.4 \%$ alginate instead of $3.3 \%$ alginate and $0.05 \%$ polylysine instead of $0.1 \%$ polylysine. This higher degree of permeability allows for faster exchange of water and the dehydrant over the capsule membrane and is thus associated with less distortion. By adding the gradual dehydration steps, the GMA procedure can be applied in the absence of any obvious distortion to capsules with low and high permeability and most importantly to capsules with immunoprotective properties. We find GMA embedding to be the best option for processing of tissues encapsulated in hydrophilic membranes. It is not associated with any obvious histological artifacts and may also be adequate for hydrated tissues such as lung (12), embryonic, or those in hydrophilic membranes.

\section{ACKNOWLEDGMENT}

This work was supported by a grant from the Dutch organization for scientific research to P.D.V.

\section{REFERENCES}

1.Aebischer, P., M. Goddard, A.P. Signore, and R.L. Timpson. 1994. Functional recovery in hemiparkinsonian primates transplanted with polymer-encapsulated PC12 cells. Exp. Neurol. 126:151-158.

2.Aebischer, P., P.C. Russell, L. Christenson, G. Panol, J.M. Monchik, and P.M. Galletti. 1986. A bioartificial parathyroid. ASAIO Trans. 32:134-137.

3.De Vos, P., B.J. De Haan, and R. Van Schilfgaarde. 1997. Upscaling the production of encapsulated islets. Biomaterials 18:1085-1090.

4.De Vos, P., B.J. De Haan, G.H.J. Wolters, J.H. Strubbe, and R. Van Schilfgaarde. 1997. Improved biocompatibility but limited graft survival after purification of alginate for microencapsulation of pancreatic islets. Diabetologia 40:262-270.

5.De Vos, P., B.J. De Haan, G.H.J. Wolters, and R. Van Schilfgaarde. 1996. Factors influencing the adequacy of microencapsulation of rat pancreatic islets. Transplantation 62:888-893.

6.De Vos, P., J.F. van Straaten, A.G. Nieuwenhuizen, M. de Groot, R.J. Ploeg, B.J. De Haan, and R. Van Schilfgaarde. 1999. Why do microencapsulated islet grafts fail in the absence of fibrotic overgrowth? Diabetes 48:1381-1388.

7.Fritschy, W.M., P. De Vos, H. Groen, F.A. Klatter, A. Pasma, G.H. Wolters, and R. Van Schilfgaarde. 1994. The capsular overgrowth on microencapsulated pancreatic islet grafts in streptozotocin and autoimmune diabetic rats. Transpl. Int. 7:264-271.

8.Fritschy, W.M., P.O. Gerrits, G.H. Wolters, A. Pasma, and R. Van Schilfgaarde. 1995. Glycol methacrylate embedding of alginatepolylysine microencapsulated pancreatic islets. Biotech. Histochem. 70:188-193.

9.Fritschy, W.M., G.H. Wolters, and R. Van Schilfgaarde. 1991. Effect of alginate-polylysine-alginate microencapsulation on in vitro insulin release from rat pancreatic islets. Diabetes 40:37-43.

10.Goosen, M.F.A., G.M. O'Shea, H.M. Gharapetain, S. Chou, and A.M. Sun. 1985. Optimization of microencapsulation parameters: semipermeable microcapsules as a bioartificial pancreas. Biotechnol. Bioeng. 27:146150.

11.Koo, J. and T.S.M. Chang. 1993. Secretion of erythropoietin from microencapsulated rat kidney cells. Int. J. Artif. Organs 16:557-560.

12.Leu, F.J., C.F. Chen, and A.M. Sun. 1993. A new method of tissue processing that causes no shrinkage or distortion. Lab. Invest. 69:121-130.

13.Lim, F. and A.M. Sun. 1980. Microencapsulated islets as bioartificial endocrine pancreas. Science 210:908-910.

14.Lum, Z.P., M. Krestow, I.T. Tai, I. Vacek, and A.M. Sun. 1992. Xenografts of rat islets into diabetic mice. Transplantation 53:11801183.

15.Nathan, D.M. 1996. The pathophysiology of diabetic complications: how much does the glucose hypothesis explain? Ann. Intern. Med. 124:86-89.

16.Soon Shiong, P., E. Feldman, R. Nelson, R.
Heintz, Q. Yao, Z. Yao, T. Zheng, N. Merideth et al. 1993. Long-term reversal of diabetes by the injection of immunoprotected islets. Proc. Natl. Acad. Sci. USA 90:58435847

17.Sun, Y.L., X.J. Ma, D.B. Zhou, I. Vacek, and A.M. Sun. 1996. Normalization of diabetes in spontaneously diabetic cynomologus monkeys by xenografts of microencapsulated porcine islets without immunosuppression. J. Clin. Invest. 98:1417-1422.

18. Van Suylichem, P.T., G.H. Wolters, and R. Van Schilfgaarde. 1990. The efficacy of density gradients for islet purification: a comparison of seven density gradients. Transpl. Int. 3:156-161.

19.Wolters, G.H., P.T. van Suylichem, J.H. Van Deijnen, and R. Van Schilfgaarde. 1990. Factors influencing the isolation process of islets of Langerhans. Horm. Metab. Res. Suppl. 25:20-26.

20.Wolters, G.H.J., W.M. Fritschy, D. Gerrits, and R. Van Schilfgaarde. 1992. A versatile alginate droplet generator applicable for $\mathrm{mi}$ croencapsulation of pancreatic islets. J. Appl. Biomater. 3:281-286.

21.Wong, H. and T.M. Chang. 1986. Bioartificial liver: implanted artificial cells microencapsulated living hepatocytes increases survival of liver failure rats. Int. J. Artif. Org. 9:335-336

Received 4 June 2001; accepted 10 September 2001.

Address correspondence to:

Dr. Paul De Vos

P/a Clinical Immunology

Pathology and Laboratory Medicine

Section of Medical Biology

University of Groningen

Hanzeplein 1

9700 RB Groningen

The Netherlands

e-mail:p.de.vos@med.rug.nl

For reprints of this or any other article, contact Reprints@BioTechniques.com 